Notre Dame Journal of Formal Logic

Volume XIII, Number 4, October 1972

NDJFAM

\title{
NATURAL DEDUCTION RULES FOR MODAL LOGICS
}

\author{
THOMAS W. SATRE
}

Natural deduction rules for systems of modal logic have been formulated in the style of Gentzen by Ohnishi, Matsumoto, Kanger, and Curry. The purpose of this paper is to formulate natural deduction rules for the S-, E-, and D-families of systems studied by Lemmon in [6]. Some of the rules for modal operators contained in this paper have been employed by others. The remainder are, to the author's knowledge, new. The style of presentation of the rules shall be that of Lemmon's natural deduction rules for propositional calculus in [5]; similar formulations of rules are to be found in [15]. In section 1 the standard proof of the deduction theorem is shown to hold in the three families of systems to be studied. In section 2 each system of these families is given a deductively equivalent formalization by means of natural deduction rules. Finally, some suggestions concerning interpretation of modal logics are offered in section 3.

1. In [6] Lemmon showed that the Lewis systems S1-S5, together with S0.5, S0.9, and the families of systems D1-D5 and E1-E5, can be axiomatized as extensions of classical propositional calculus. The propositional calculus basis common to all these systems shall be referred to as $\mathrm{PC}$ and shall have the axioms and rules:

PC1: $\quad p \supset(q \supset p)$

PC2: $\quad(p \supset(q \supset \gamma)) \supset((p \supset q) \supset(p \supset r))$

PC3: $\quad(-q \supset-p) \supset((-q \supset p) \supset q)$

PC4: If $\vdash A$ and $B$ comes from $A$ by uniform substitution for propositional variables of $A$, then $\vdash B$.

PC5: From $A$ and $A \supset B, B$ may be inferred.

The usual definitions of $\&, v$, and $\equiv$ are assumed. Further, it is assumed that the reader is acquainted with the modal rules (a)-(D), the modal axioms (1)-(5), and the definitions of $\leftrightarrow, \diamond$, and $\equiv$ of $[6] .^{1}$

1. In the notation of [6] these are respectively $C^{\prime}, M$, and $E^{\prime}$. The primitive modal operator there is $L$ which is represented by $\square$ in this paper. 
To illustrate the deduction theorem for systems of modal logic let $\mathrm{K}$ be the system obtained by adding to $P C$ axiom $\left(1^{\prime}\right)$ and rule (a). The definition of a wff of PC is assumed to be extended to cover wffs of modal logic. ${ }^{2}$ The standard definition of proof in a system $S$ from hypotheses is adopted here, viz., a wff $B$ is provable in $S$ from hypotheses $A_{1}, \ldots, A_{n}$ (written $A_{1}, \ldots, A_{n} \vdash B$ in $\left.S\right)$ if and only if there is a sequence of wffs $B_{1}, \ldots, B_{m}$ such that $B_{m}=B$ and for each $i(1 \leq i \leq m)$ either:

(i) $B_{i}$ is an axiom of $S$; or

(ii) $B_{i}$ is one of $A_{1}, \ldots, A_{n}$; or

(iii) $B_{i}$ follows directly from some $B_{j_{1}}, \ldots, B_{j_{k}}\left(1 \leq j_{k}<i\right)$ by some rule of $S$.

Where $n=0, B$ is said to be a theorem of $S$ (written $\vdash B$ in $S$ ). The use of ' $\vdash$ ' here thus corresponds to that of Kleene in [4], p. 88. It is a property of the notion of proof from hypotheses that if $\vdash B$ in $S$ then, trivially, $A_{1}, \ldots$, $A_{n} \vdash B$ in $S$. This property is used without mention in the proofs that follow.

Deduction theorem for K. If $A_{1}, \ldots, A_{n} \vdash B$ in $\mathrm{K}$, then $A_{1}, \ldots, A_{n-1} \vdash A_{n} \supset$ $B$ in $\mathrm{K}$.

Proof: By supposition and the definition of proof from hypotheses, there is a sequence $B_{1}, \ldots, B_{m}$ of wffs such that for each $B_{i}(1 \leq i \leq m)$ either (i) $B_{i}$ is some $A_{j}(1 \leq j \leq n)$; or (ii) $B_{i}$ is one of PC1-PC 3 , or (1'); or (iii) $B_{i}$ comes from $B_{j}(j<i)$ by PC4; or (iv) $B_{i}$ comes from some $B_{j}$ and $B_{k}=\left(B_{j} \supset B_{i}\right)(j, k<i)$ by PC5; or (v) $B_{i}$ is $\square B_{j}$ and comes from $B_{j}$ by rule (a). By proving the theorem for all $i \leq m$, the result follows by putting $i=m$.

Case (i) (a): If $j=n$, then $B_{i}$ is $A_{n}$. But $\vdash A_{n} \supset A_{n}$ in $\mathrm{K}$ in virtue of PC. So $\vdash A_{n} \supset B_{i}$ in $\mathrm{K}$ from which it follows that $A_{1}, \ldots, A_{n-1} \vdash A_{n} \supset B_{i}$ in $\mathrm{K}$. (b): If $j \neq n$, then, since $\vdash A_{j} \supset A_{j}$ in $\mathrm{K}$, we have $A_{1}, \ldots, A_{n-1} \vdash A_{j}$ in $\mathrm{K}$ which is $A_{1}, \ldots, A_{n-1} \vdash B_{i}$ in K. By PC1 and PC5 it follows that $A_{1}, \ldots$, $A_{n-1} \vdash A_{n} \supset B_{i}$ in $\mathrm{K}$.

Case (ii): By definition $\vdash B_{i}$ in $\mathrm{K}$. By appropriate substitution in PC1 we have $\vdash B_{i} \supset\left(A_{n} \supset B_{i}\right)$ in K. Thus $\vdash A_{n} \supset B_{i}$ in K by PC5. Hence, $A_{1}, \ldots$, $A_{n-1} \vdash A_{n} \supset B_{i}$ in $\mathrm{K}$.

Case (iii): Then, by the restriction on PC4, it follows that $\vdash B_{i}$ in K. So $A_{1}, \ldots, A_{n-1} \vdash A_{n} \supset B_{i}$ in $\mathrm{K}$ by the same reasoning as case (ii).

Case (iv): As inductive hypotheses, suppose that the theorem holds for $B_{j}$ and $B_{k}$. Thus, we have both $A_{1}, \ldots, A_{n-1} \vdash A_{n} \supset B_{j}$ in $\mathrm{K}$ and $A_{1}, \ldots$, $A_{n-1} \vdash A_{n} \supset\left(B_{j} \supset B_{i}\right)$ in K. As a case of $\mathrm{PC} 2 \vdash\left(A_{n} \supset\left(B_{j} \supset B_{i}\right)\right) \supset\left(\left(A_{n} \supset B_{j}\right) \supset\right.$ $\left.\left(A_{n} \supset B_{i}\right)\right)$ in K. Two steps of PC4 yield $A_{1}, \ldots, A_{n-1} \vdash A_{n} \supset B_{i}$ in K.

Case (v): By the restriction on rule (a) we have $\vdash B_{j}$ in $\mathrm{K}$, and by rule

2. The system $\mathrm{K}$, as formulated here, was presented by Lemmon in lectures during the spring term of 1966. 
(a) $\vdash \square B_{j}$ in $\mathrm{K}$ which is $\vdash B_{i}$ in $\mathrm{K}$. Again, the proof is the same as that in case (ii).

It will be noted that cases (i)-(iv) give just the standard proof of the deduction theorem for classical propositional calculus (vide [13], pp. 16-18). Case (iii) could be omitted if PC1-3 had been stated as axiom schemata. However, PC4 is needed when we consider the addition of modal operators, with appropriate axioms and rules, to PC. The addition of the modal axiom ( $\left.1^{\prime}\right)$ made no difference at case (ii), and no restrictions are needed on the deduction theorem due to rule (a) since the rule will apply only to theorems.

The deduction theorem, as well as rules (a)-(Eb'), is a subsidiary deduction rule, in the sense of Kleene [4], \$22. It is not in general true that a subsidiary deduction rule will continue to hold when a system is extended by addition of new axioms or rules, since there may not be resulting deductions available in the extended system. Examination of the above proof will show that the restriction on rule (a) provides that the only new subsidiary deductions will be of theorems; thus, the corresponding resulting deductions will always be available in virtue of PC1, 2, 4, and 5 . (PC3 was not used in the proof of the deduction theorem for K.)

We can generalize the result obtained as follows: Let $S$ be any formal system having the symbols and formation rules of PC and obtained by adding to PC proper axioms and rules, together with formation rules for any new symbols introduced in the proper axioms and rules. Then the deduction theorem above will hold for $S$ provided that each proper rule is applicable only to theorems of $S$, i.e., formulas provable from zero hypotheses. ${ }^{3}$ It will be observed that all of the proper rules (a)-(D) are of this type. The restrictions on $\left(\mathrm{Eb}^{\prime}\right)$ and $(\mathrm{D})$ concern only the presence or absence of modal operators in component wffs of the theorems which result, and $\left(a^{\prime}\right)$ can easily be stated so as to show that it applies only to theorems of any system to which it is added. Hence, we may conclude that the deduction theorem above holds for all of Lemmon's S-, E-, and D-systems.

2. As a basic set of natural deduction rules the following, taken from Lemmon [5], shall be used:

Hyp: Any wff may be introduced at any line of a proof.

MP: $\quad$ Given $A$ and $A \supset B$, we may derive $B$.

$\mathrm{DN}$ : Given $A$, we may derive - $-A$, and vice versa.

\&I: $\quad$ Given $A$ and $B$, we may derive $A \& B$.

\&E: $\quad$ Given $A \& B$, we may derive either $A$ or $B$ separately.

vI: $\quad$ Given either $A$ or $B$ separately, we may derive $A \vee B$.

CP: Given a proof of $B$ depending upon $A$ as a hypothesis, we may derive $A \supset B$ depending upon the remaining hypotheses.

3. In [8], p. 58, it is noted that the deduction theorem will continue to hold in extensions of PC formed by the addition of new axioms alone. 
$\vee E$ : Given $A \vee B$ together with a proof of $C$ from $A$ as a hypothesis and a proof of $C$ from $B$ as a hypothesis, we may derive $C$ depending upon any hypotheses upon which $A \vee B$ depends or upon which $C$ depends in its derivation from $A$ (except for $A$ ) or upon which $C$ depends in its derivation from $B$ (except for $B$ ).

RAA: Given a proof of $B \&-B$ depending upon $A$ as a hypothesis, we may derive $-A$ depending upon the remaining hypotheses.

In the case of rule Hyp, the conclusion derived depends upon itself as a hypothesis. For the rules MP-vI, the conclusion depends upon the combined hypotheses (if any) of the premise(s). For the remaining rules, the conclusion rests upon the hypotheses stipulated by the rule. Schematically, these rules may be written as follows: let $\Gamma, \Delta$ be sequences (possibly empty) of wffs, or conjunctions (possibly empty) of wffs, according as $\Gamma, \Delta$ occur to the left or right side of ' $\vdash$ '. To the left of ' $\vdash$ ' occur only hypotheses, if any, from which the wff occurring to the right is said to be deducible. Wffs to the right of ' $\vdash$ ' and above a horizontal line are premises from which the wff to the right of ' $\vdash$ ' and below the horizontal line is derivable in accordance with the rule in question. To the left of ' - ' and below the horizontal line are the hypotheses upon which a conclusion drawn in accordance with the rule will depend. By rule Hyp any hypothesis is also available as a premise in a proof, though, of course, a premise need not have been introduced by the rule Hyp.

$$
\begin{aligned}
& \text { Hyp: } A \vdash A \\
& \mathrm{MP}: \frac{\Gamma \vdash A ; \Delta \vdash A \supset B}{\Gamma, \Delta \vdash B} \\
& \mathrm{RAA}: \frac{A_{1}, \ldots, A_{n} \vdash B \&-B}{A_{1}, \ldots, A_{j-1}, A_{j+1}, \ldots, A_{n} \vdash-A_{j}(1 \leq j \leq n)} \\
& \& \mathrm{I}: \frac{\Gamma \vdash A ; \Delta \vdash B}{\Gamma, \Delta \vdash A \& B} \\
& \mathrm{CP}: \frac{A_{1}, \ldots, A_{n} \vdash B}{A_{1}, \ldots, A_{j-1}, A_{j+1}, \ldots, A_{n} \vdash A_{j} \supset B(1 \leq j \leq n)} \\
& \& \mathrm{E}: \quad \frac{\Gamma \vdash A \& B}{\Gamma \vdash A} \frac{\Gamma \vdash A \& B}{\Gamma \vdash B} \\
& \vee \mathrm{I}: \frac{\Gamma \vdash A}{\Gamma \vdash A \vee B} \frac{\Delta \vdash B}{\Delta \vdash A \vee B} \vee \mathrm{E}: \frac{\Gamma \vdash A \vee B ; \Delta, A \vdash C ; \Delta^{\prime}, B \vdash C}{\Gamma, \Delta, \Delta^{\prime} \vdash C} \\
& \mathrm{DN}: \frac{\Gamma \vdash A}{\Gamma \vdash-}-A \quad \frac{\Gamma \vdash--A}{\Gamma \vdash A}
\end{aligned}
$$

For convenience, the rule of substitution for propositional variables shall be taken over for natural deduction also, though it could be derived as well. The above rules, together with the rule of substitution for propositional variables, will be referred to as NPC.

In this section systems of modal logic are constructed by adding various rules to NPC. The result will be families of systems deductively equivalent to the S-, E-, and D-families and sharing NPC as a basis just as those systems share PC as a basis on Lemmon's formalization. The deductively equivalent natural deduction systems will be indicated by prefixing ' $\mathrm{N}$ ' to the name of the system. As a stock of natural deduction rules for modal operators the following are adopted: 
$\square \mathrm{E}_{1}$ : Given $\square A$ we may derive $A$ depending upon the same hypotheses as the premise.

$\square \mathrm{E}_{2}$ : Given $\square A$ we may derive $\diamond A$ depending upon the same hypotheses as the premise.

$\square \mathrm{E}_{3}$ : Given $\square A$ we may derive separately $\diamond A$, or, if $A$ is fully modalized, $A$. The conclusion depends upon the same hypotheses as the premise. (A wff is said to be fully modalized if each occurrence of a propositional variable within it is within the scope of some modal operator also within it.)

Let $\Gamma, \Delta$ be any sequences (possibly empty) of wffs, or conjunctions of wffs as before. Let $\square \Delta$ be the result of prefixing ' $\square$ ' to each wff of $\Delta$.

$\square \mathrm{I}_{1}$ : Given $\square \Delta$ and a proof of $B$ from $\Delta$ alone as hypotheses, we may derive $\square B$ depending upon the combined hypotheses of the premises $\square \Delta$.

$\square \mathrm{I}_{2}$ : Given $\square \Delta$ and a proof of $B$ from $\Delta$ alone as hypotheses, we may derive $\square B$, provided that $\Delta$ is not empty. The conclusion depends upon the combined hypotheses of the premises $\square \Delta$.

$\square I_{3}$ : Given $\square \Delta$ and a proof of $B$ from $\Delta$ alone as hypotheses, we may derive $\square B$, provided that $\Delta$ is not empty and that all wffs of $\Delta$ and $B$ are wffs of propositional calculus, i.e., $\Delta, B$ contain no occurrences of modal operators. The conclusion depends upon the combined hypotheses of the premises $\square \Delta$.

$\square \mathrm{I}_{4}$ : Given $B$ we may derive $\square B$ depending upon the same hypotheses, provided that each of those hypotheses is of the form $\square C$ for some wff $C$.

$\square \mathrm{I}_{5}$ : Given $B$ depending upon some hypotheses, we may derive $\square B$, provided that each of those hypotheses is of the form $\square C$ for some wff $C$. The conclusion depends upon the same hypotheses as the premise.

$\square \mathrm{I}_{6}$ : Given $B$ we may derive $\square B$, depending upon the same hypotheses, provided that each of those hypotheses is of the form of either $\square C$ or $\diamond C$ for some wff $C$.

$\square \mathrm{I}_{7}$ : Given $B$ depending upon some hypotheses, we may derive $\square B$, provided that each of those hypotheses is of the form of either $\square C$ or $\diamond C$ for some wff $C$. The conclusion depends upon the same hypotheses as the premise.

$\square \mathrm{I}_{8}$ : (Rule Scheme) Given $\square \Delta$, where $\Delta$ is not empty, and a proof of $B$ by rules of NPC alone from $\Delta$ alone as hypotheses, we may derive $\square B$, depending upon the combined hypotheses of the premises; and, if $\Delta$ is empty and $\vdash B$, then $\vdash \square B$, provided that $B$ is in $\Lambda$.

$\square I_{9}$ : Given $\square \Delta$ as premises and a proof of $B$ by rules NPC alone from $\Delta$ alone as hypotheses, we may derive $\square B$ depending upon the same hypotheses as the premises $\square \Delta$.

$\square \mathrm{I}_{10}$ : Given a proof of $B$ from $\Delta$ alone as hypotheses, where $\Delta$ is not empty, then (i) given as premises $\square \Delta$ we may derive $\square B$ depending upon the hypotheses upon which $\square \Delta$ depend; and (ii) if each hypothesis of $\Delta$ is of the form $\square C$, for some wff $C$, then we may derive $\square B$ depending upon $\Delta$.

$\square \mathrm{I}_{11}$ : Given a proof of $B$ from $\Delta$ alone as hypotheses, where $\Delta$ is not empty, then (i) given as premises $\square \Delta$ we may derive $\square B$ depending upon 
the hypotheses upon which $\square \Delta$ depend; and (ii) if each hypothesis of $\Delta$ is of the form of either $\square C$, or $\diamond C$, for some wff $C$, then we may derive $\square B$ depending upon $\Delta$.

$\square \mathrm{S}_{1}$ : Given as a premise $\square(A \supset B)$ we may derive $\square(\square A \supset \square B)$ depending upon the same hypotheses, if any.

$\square \mathrm{S}_{2}$ : Given a proof of $\square(A \supset B)$ as a theorem, i.e., $\vdash \square(A \supset B)$, we may derive as a theorem $\square(\square A \supset \square B)$.

$\square \mathrm{S}_{3}$ : Given proofs of $\square(A \supset B)$ and $\square(B \supset A)$ as theorems, we may derive $\square(\square A \supset \square B)$ as a theorem.

These rules may be presented schematically as follows:

$\square \mathrm{E}_{1}: \frac{\Gamma \vdash \square A}{\Gamma \vdash A}$.

$\square \mathrm{E}_{3}: \frac{\Gamma \vdash \square A}{\Gamma \vdash \diamond A}$; and $\frac{\Gamma \vdash \square A}{\Gamma \vdash A}$, provided $A$ is fully modalized.

$\square \mathrm{I}_{1}: \frac{\Gamma \vdash \square \Delta ; \Delta \vdash B}{\Gamma \vdash \square B} . \quad \square \mathrm{I}_{2}: \frac{\Gamma \vdash \square \Delta ; \Delta \vdash B}{\Gamma \vdash \square B}$, provided $\Delta$ is not empty.

$\square \mathrm{I}_{3}: \frac{\Gamma \vdash \square \Delta ; \Delta \vdash B}{\Gamma \vdash \square B}$, provided that $\Delta$ is not empty and that no wffs $\Delta$ or $B$ contain occurrences of modal operators.

$\square \mathrm{I}_{4}: \frac{\Delta \vdash B}{\Delta \vdash \square B}$, provided that each wff of $\Delta$ is of the form $\square C$ for some

$\square I_{5}: \frac{\Delta \vdash B}{\Delta \vdash \square B}$, provided that $\Delta$ is not empty and that each wff of $\Delta$ is of the form $\square C$ for some wff $C$.

$\square \mathrm{I}_{6}: \frac{\Delta \vdash B}{\Delta \vdash \square B}$, provided that each wff of $\Delta$ is of the form of either $\square C$
or $\Delta C$ for some wff $C$. $\square \mathrm{I}_{7}: \frac{\Delta \vdash B}{\Delta \vdash \square B}$, provided that $\Delta$ is not empty and that each wff of $\Delta$ is of

$\square \mathrm{I}_{8}: \quad \frac{\Gamma \vdash \square \Delta ; \Delta \vdash B \text { in NPC }}{\Gamma \vdash \square B}$, provided that $\Delta$ is not empty, and $\frac{\vdash B}{1 \square B}$, provided that $B$ is in $\Lambda$.

$\square \mathrm{I}_{9}: \quad \frac{\Gamma \vdash \square \Delta ; \Delta \vdash B \text { in NPC }}{\Gamma \vdash \square B}$. $\square \mathrm{I}_{10}: \frac{\Gamma \vdash \square \Delta ; \Delta \vdash B}{\Gamma \vdash \square B}$, and $\frac{\Delta \vdash B}{\Delta \vdash \square B}$, provided that all wffs of $\Delta$ are of the case $\Delta$ is not empty. $\square \mathrm{I}_{11}: \frac{\Gamma \vdash \square \Delta ; \Delta \vdash B}{\Gamma \vdash \square B}$, and $\frac{\Delta \vdash B}{\Delta \vdash \square B}, \begin{aligned} & \text { provided that all hypotheses of } \Delta \text { are } \\ & \text { of the form of either } \square C \text { or } \diamond C \text { for }\end{aligned}$ some wff $C$; in either case $\Delta$ is not empty.

$$
\begin{array}{ll}
\square \mathrm{S}_{1}: & \frac{\Gamma \vdash \square(A \supset B)}{\Gamma \vdash \square(\square A \supset \square B)} . \\
\square \mathrm{S}_{3}: & \frac{\vdash \square(A \supset B) ; \vdash \square(B \supset A)}{\vdash \square(\square A \supset \square B)} .
\end{array}
$$$$
\square \mathrm{S}_{2}: \frac{\vdash \square(A \supset B)}{\vdash \square(\square A \supset \square B)} .
$$ 
The motive behind the formalization here is to provide as constant factors in all of the theories to be studied the classical propositional calculus as well as the notion of deduction in accordance with rules alone. The differences between modal systems are shown by differences only in the rules governing modal operators. This is just what Lemmon-style formalization does from an axiomatic approach. A rule much like $\square \mathrm{I}_{1}$ was suggested by Lemmon in lectures in 1966. From it a rule like Ohnishi and Matsumoto's $\rightarrow \square$ for $M^{*}$ in [10] could be obtained given appropriate translation from Gentzen-style notation. $\square \mathrm{I}_{2}$ is likewise related to rule $\rightarrow \square$ for system Q2 of [10]. $\square \mathrm{I}_{4}$ is essentially rule $\rightarrow \square$ for S4* of [10], and $\square \mathrm{I}_{5}$ is just $\square \mathrm{I}_{4}$ with the restriction that $\Delta$ ( $\square \theta$ in [10]) not be empty. $\square \mathrm{I}_{8}$ should be compared with Ohnishi's rules $\rightarrow \square$ for $\mathrm{S} 2 *$ and $\mathrm{S} 3 *$ of [9]. Ohnishi's use of 'tautology' there is not exactly that of Lemmon in [6], and Ohnishi's restriction, p. 126 of [9], does not seem to restrict use of his rule $\mathrm{RT}$ when $\alpha$ is a theorem other than an instance of a tautology of propositional calculus or an instance of (1') or (2). $\square \mathrm{I}_{8}$ obtains these restrictions by a class of wffs $\Lambda$, fixed for a given system, and by the restriction that $B$ be derived from $\Delta$ by NPC alone. Ohnishi obtains a counterpart of the latter restriction by not allowing rule $\rightarrow \square$ to occur below $R T$ in a proof figure. When applied in system $S 3, \square I_{8}$ seems easier to employ than would be a counterpart of Ohnishi's $\rightarrow \square$ for $S 3 *$ of $[9] . \square I_{9}$ and its use in NS0.5 should be compared with Routley's rule in [14] for *S0.5, which is a Gentzen-style calculus. Finally, $\square \mathrm{E}_{1}$ is a counterpart of $\square \rightarrow$ of $[10]$.

Let NK be NPC with the added rule $\square I_{1}$. The deductive equivalence of $\mathrm{K}$ and $\mathrm{NK}$, i.e., $\vdash A$ in $\mathrm{NK}$ if and only if $\vdash A$ in $\mathrm{K}$, is proven as follows: suppose that $\vdash A$ in NK. The rules of NK hold in $\mathrm{K}$.

Case (1): The rules of NPC hold in $\mathrm{K}$ in virtue of $\mathrm{PC}$ and the deduction theorem for $\mathrm{K}$.

Case (2): Rule $\square I_{1}$. If $\Delta$ is empty, in which case $\Gamma$ is also, then $\square I_{1}$ is just rule (a) of $\mathrm{K}$. So let $\Delta$ be $A_{1}, \ldots, A_{n}$ and suppose that $A_{1}, \ldots, A_{n} \vdash B$ in $\mathrm{K}$. By the deduction theorem for $\mathrm{K}$, applied $n$ times, $\vdash A_{1} \supset\left(\ldots \supset\left(A_{n} \supset\right.\right.$ $B) . .$.$) in \mathrm{K}$. By (a), (1'), and $\mathrm{PC}$ we have $\vdash \square A_{1} \supset\left(. . \supset\left(\square A_{n} \supset \square B\right) \ldots\right)$ in $\mathrm{K}$. Hence, if $\Gamma$ is any set of hypotheses such that $\Gamma \vdash \square \Delta$ in $\mathrm{K}$, then the proof of $\Gamma \vdash \square \Delta$ in $\mathrm{K}$ may be extended by use of $\mathrm{PC}$ together with the above theorem to yield a proof of $\Gamma \vdash \square B$ in $\mathrm{K}$.

Conversely, suppose that $\vdash A$ in $\mathrm{K}$. It suffices to show that the axioms and rules of $\mathrm{K}$ are derivable in NK. The axioms and rules of $\mathrm{PC}$ are in NPC and so are in NK; so, Case (1) is proven.

Case (2): Let $A$ be $\left(1^{\prime}\right)$. In NK we have the proof:

$\begin{array}{rl}1(1) \square(p \supset q) & \text { Hyp } \\ 2(2) \square p & \text { Hyp } \\ 3(3) \quad p \supset q & \text { Hyp } \\ 4(4) \quad p & \text { Hyp } \\ 3,4(5) \quad q & 3,4 \mathrm{MP} \\ 1,2(6) \square q & 1,2(=\square \Delta=\Gamma), 3,4(=\Delta), \square \mathrm{I}_{1}\end{array}$




$$
\begin{array}{rl}
1(7) \square p \supset \square q & 2,6 \mathrm{CP} \\
(8) \square(p \supset q) \supset(\square p \supset \square q) & 1,7 \mathrm{CP}
\end{array}
$$

Case (3): Let $A$ be derived by rule (a). Since rule (a) is just the special case of $\square I_{1}$ where $\Delta$ is empty, $\vdash A$ in NK. So, K and NK are deductively equivalent. $^{4}$ We may now easily obtain the natural deduction systems corresponding to $\mathrm{T}, \mathrm{S} 4$, and $\mathrm{S} 5$, which are extensions of $\mathrm{K}$, as extensions of NK. Corresponding to Lemmon's formulations of these systems we have:

T: PC; (a); (1'); (2).

S4: PC; (a); (1'); (2); (4).

S5: ${ }^{5} \mathrm{PC} ;(\mathrm{a}) ;\left(1^{\prime}\right) ;(2) ;(5)$.
NT: NPC; $\square \mathrm{E}_{1} ; \square \mathrm{I}_{1}$.

NS4: NPC; $\square \mathrm{E}_{1} ; \square \mathrm{I}_{4}$.

NS5: NPC; $\square \mathrm{E}_{1} ; \square \mathrm{I}_{6}$.

Since NT (T) is just NK (K) with the added rule (axiom) $\square \mathrm{E}_{1}(\operatorname{axiom}(2)$ ), the deductive equivalence of $\mathrm{NT}$ and $\mathrm{T}$ is proven by the previous proof except that we now add cases for $\square \mathrm{E}_{1}$ and (2). From (2) of $\mathrm{T}$ we easily derive $\square \mathrm{E}_{1}$, since, given $\square A$ as a premise, $A$ always follows depending upon the same hypotheses by appropriate substitution on axiom (2) and MP. Conversely, given $\square \mathrm{E}_{1}$ of NT, (2) is proven as follows:

$$
\begin{array}{rl}
1(1) \square p & \text { Hyp } \\
1(2) p & 1, \square \mathrm{E}_{1} \\
\text { (3) } \square p \supset p & 1,2 \mathrm{CP}
\end{array}
$$

Thus, NT and $\mathrm{T}$ are deductively equivalent. Since $\square \mathrm{E}_{1}$ and (2) are equivalent in the presence of NPC or PC, proofs of this equivalence will be omitted in what follows.

To establish the deductive equivalence of NS4 and S4 it suffices to show that $\square \mathrm{I}_{4}$ is deductively equivalent to (a), (1'), and (4) in the presence of NPC and $\square \mathrm{E}_{1}$ (alternatively, in the presence of $P C$ and (2)). Given (a), (1'), and (4), $\square \mathrm{I}_{4}$ is derived as follows: if $\vdash B$ in $\mathrm{S} 4$, then $\vdash \square B$ in $\mathrm{S} 4$ by rule (a). So suppose that $\Delta$ is not empty, i.e., $A_{1}, \ldots, A_{n} \vdash B$ in $\mathrm{S} 4$ where each $A_{i}(1 \leq i \leq n)$ is $\square C_{i}$ for some wff $C_{i}$. By the deduction theorem for S4 and PC we have $\vdash\left(A_{1} \& \ldots \& A_{n}\right) \supset B$ in S4 which, by supposition, is $\vdash\left(\square C_{1} \& \ldots \& \square C_{n}\right) \supset B$ in $\mathrm{S} 4$. By the theorem of $\mathrm{S} 4$ that $\left(\square C_{1} \& \ldots\right.$ $\left.\& \square C_{n}\right) \equiv \square\left(C_{1} \& \ldots \& C_{n}\right)$ and substitution of material equivalents we have $\vdash \square\left(C_{1} \& \ldots \& C_{n}\right) \supset B$ in S4. By (a) and (1') this yields $\vdash \square \square\left(C_{1} \& \ldots\right.$ $\left.\& C_{n}\right) \supset \square B$ in $\mathrm{S} 4$, from which, by $\mathrm{PC}$ and appropriate substitution on axiom (4), we have $\vdash \square\left(C_{1} \& \ldots \& C_{n}\right) \supset \square B$ in $\mathrm{S} 4$. By the previously used equivalence we have $\vdash\left(\square C_{1} \& \ldots \& \square C_{n}\right) \supset \square B$ in $\mathrm{S} 4$ which is $\vdash\left(A_{1} \& \ldots\right.$

4. Case 2 of this proof also illustrates the formal proof procedure in NK. Aiming to prove $\left(1^{\prime}\right)$ one tries to prove $\square q$ from $\square(p \supset q)$ and $\square p$ as hypotheses. The strategy for employing $\square \mathrm{I}_{1}$ is to add as hypotheses $p \supset q$ and $p$ and then prove $q$. In step (6) of $\square \mathrm{I}_{1}, p \supset q$ and $p$ are discharged as hypotheses, and the conclusion is put to depend upon hypotheses of $\square(p \supset q)$ and $\square p$, which here happen to be themselves. The numbers at the extreme left provide a record of the hypotheses upon which any given line in the proof depends.

5. This formulation of S5 is taken from Prior, [12], who attributes it to Gödel. 
$\left.\& A_{n}\right) \supset \square B$ in $\mathrm{S} 4$. Hence, given as premises in a proof $A_{1}, \ldots, A_{n}$ depending upon $\Gamma$, the last theorem together with PC yields a proof of $\Gamma \vdash \square B$ in S4. Conversely, given $\square \mathrm{I}_{4}\left(1^{\prime}\right)$ is proven in NS4 by the same proof given in NK since $\square \mathrm{I}_{1}$ is easily derived from $\square \mathrm{I}_{4}$ in the presence of $\square \mathrm{E}_{1}$. For (4) we have the proof in NS4:

$$
\begin{array}{ll}
1 \text { (1) } \square p & \text { Hyp } \\
1 \text { (2) } \square \square p & 1, \square \mathrm{I}_{4} \\
\text { (3) } \square p \supset \square \square p & 1,2 \mathrm{CP} .
\end{array}
$$

Note that ' $\square p$ ' here is the appropriate $B$ as well as the one member sequence $\Delta$; so the step at (2) is a correct use of $\square \mathrm{I}_{4}$. For rule (a), it suffices to note that (a) is just $\square \mathrm{I}_{4}$ for the case where $B$ depends upon no hypotheses. So NS4 and S4 are equivalent.

The deductive equivalence of NS5 and S5 is proven similarly by showing that $\square \mathrm{I}_{6}$ is deductively equivalent to (a), (1'), and (5) in the presence of NPC and $\square E_{1}$ (alternatively, in the presence of (2) and PC). $\square \mathrm{I}_{6}$ is derived in S5 as follows: if $\Delta$ is empty, then $\square \mathrm{I}_{6}$ is just (a). Suppose that $\Delta$ is not empty,i.e., that $A_{1}, \ldots, A_{n} \vdash B$ in S5 where each $A_{i}(1 \leq i \leq n)$ is either $\square C_{i}$ or $\diamond C_{i}$ for some wff $C_{i}$. By the deduction theorem for S5: $\vdash A_{1} \supset\left(\ldots \supset\left(A_{n} \supset B\right) \ldots\right)$ in S5. By $(\mathrm{a}),\left(1^{\prime}\right)$, and PC we have:

$$
\text { (i) } \vdash \square A_{1} \supset\left(\ldots \supset\left(\square A_{n} \supset \square B\right) \ldots \text {. . in } \mathrm{S} 5\right. \text {. }
$$

By appropriate substitution on (5) and the definition of $\diamond, \vdash \diamond C_{i} \supset \square \diamond C_{i}$ in S5. Further, (4) is a theorem of S5; hence, $\vdash \square C_{i} \supset \square \square C_{i}$ in S5. So, whether $A_{i}$ is $\square C_{i}$ or $\diamond C_{i}$ we have

$$
\text { (ii) } \vdash A_{i} \supset \square A_{i} \text { in S5. }
$$

By PC, (i) and (ii) yield $\vdash A_{1} \supset\left(\ldots \supset\left(A_{n} \supset \square B\right) \ldots\right)$ in S5. Thus given as premises $A_{1}, \ldots, A_{n}$ the last theorem permits the proof in S5 of $\square B$ depending upon the same hypotheses as the premises. Conversely, given $\square I_{6}, \square I_{4}$ is easily derived. Hence, the proof of $\left(1^{\prime}\right)$ in NS5 is the same as that given in NK since $\square I_{1}$ is derivable from $\square I_{4}$ in the presence of $\square E_{1}$. Rule (a) is the special case of $\square \mathrm{I}_{6}$ where $B$ is a theorem. Finally (5) is proven in NS5 as follows:

$\begin{array}{cl}1(1) \diamond-p & \text { Hyp } \\ 1(2)-\square--p & 2, \text { Df } \\ (3)-\square--p \supset-\square p & \text { Theorem of NK } \\ 1(4)-\square p & 2,3 \text { MP } \\ 1(5) \square-\square p & 4 \square \mathrm{I}_{6} \\ (6) \diamond-p \supset \square-\square p & 1,5 \text { CP } \\ (7)-\square p \supset \diamond-p & \text { Theorem of NK } \\ (8)-\square p \supset \square-\square p & 6,7 \text { by NPC }\end{array}$

Lines (3) and (7) are easily derived in NK which is contained in NS5, and their introduction as theorems in the proof can be taken as an abbreviated way of inserting complete proofs of them. Similarly, the step at line (8) can be taken as an abbreviated way of inserting a proof of (8) from lines (6) and (7). 
The expected containments hold for NK-NS5. Further, the progression from NT to NS5 can be viewed as progressive liberalizations of the rule for introducing ' $\square$ ' (in the presence of $\square \mathrm{E}_{1}$ ) into proofs from hypotheses. Given that $B$ is provable from hypotheses $A_{1}, \ldots, A_{n}, \square \mathrm{I}_{1}$ permitted one to derive $\square B$ only by strengthening his hypotheses to $\square A_{1}, \ldots, \square A_{n} . \quad \square \mathrm{I}_{4}$ liberalizes this to permit deriving $\square B$ anyway, provided that each hypothesis was of the form of $\square C$ to begin with. $\square \mathrm{I}_{6}$ further liberalizes this to the condition that each hypothesis has been of the form of either $\square C$ or $\diamond C$.

Just as we obtain extensions of NK or $\mathrm{K}$ by either liberalizing rules for ' $\square$ ' or by adding axioms, we obtain contractions of NK or $\mathrm{K}$ by either dropping axioms or restricting rules. By adopting more restricted rules for introducing ' $\square$ ' we have weaker NS- or S-systems. Corresponding to Lemmon's formalizations we have

S3: PC; (a'); (1); (2).

NS3: NPC; $\square \mathrm{E}_{1} ; \square \mathrm{I}_{8}$, where $\Lambda$ is the set of all substitution instances of tautologies, of (1), and of (2); $\square \mathrm{S}_{1}$.

S2: PC; $\left(a^{\prime}\right) ;(b) ;\left(1^{\prime}\right) ;(2)$. NS2: NPC; $\square \mathrm{E}_{1} ; \square \mathrm{I}_{8}$, where $\Lambda$ is the set of all substitution instances of tautologies, of $\left(1^{\prime}\right)$, and of (2); $\square \mathrm{S}_{2}$.

S1: PC; $\left(a^{\prime}\right) ;\left(b^{\prime}\right) ;(3) ;(2)$. NS1: NPC; $\square \mathrm{E}_{1} ; \square \mathrm{I}_{8}$, where $\Lambda$ is the set of all substitution instances of tautologies, of (3), and of $(2) ; \square \mathrm{S}_{3}$.

For the deductive equivalence of NS1 and S1 it suffices to show that $\square I_{8}$ for NS1 and $\square S_{3}$ are deductively equivalent to $\left(a^{\prime}\right)$ for $S 1,\left(b^{\prime}\right)$, and (3) in the presence of NPC and $\square E_{1}$ (or PC and (2)). $\square I_{8}$ for NS1 is proven for S1 as follows: if $\Delta$ is empty (in which case $\Gamma$ is also), then $\square I_{8}$ is just $\left(a^{\prime}\right)$ of S1. So let $\Delta$ be $A_{1}, \ldots, A_{n}$ and suppose that $A_{1}, \ldots, A_{n} \vdash B$ in NPC. By the equivalence of NPC and PC and the deduction theorem, we have:

$$
\text { (i) } \vdash A_{1} \supset\left(\ldots \supset\left(A_{n} \supset B\right) \ldots\right) \text { in PC. }
$$

Since (i) is an instance of a theorem of PC, it is an instance of a tautology. So by $\left(\mathrm{a}^{\prime}\right)$ and PC4 of $\mathrm{S} 1$ we get $\vdash \square\left(A_{1} \supset\left(\ldots \supset\left(A_{n} \supset B\right) \ldots\right)\right)$ in S1. (1') is derivable in $\mathrm{S} 1$; this together with $\mathrm{PC}$ yields

$$
\text { (ii) } \vdash \square A_{1} \supset\left(. . \supset\left(\square A_{n} \supset \square B\right) \ldots \text { ) in } \mathrm{S} 1\right. \text {. }
$$

Thus, given as premises $\square A_{1}, \ldots, \square A_{n}$ depending upon $\Gamma$, (ii) and $\mathrm{PC}$ will yield a proof of $\Gamma \vdash \square B . \square S_{3}$ is derived by $\left(b^{\prime}\right)$ and the definition of $\equiv$ by substitution on the theorem $\square(\square p \supset \square p)$ of S1. Conversely, in NS1 we prove (3) as follows:

$\begin{array}{rl}1(1) \square(p \supset q) & \text { Hyp } \\ 2(2) \square(q \supset r) & \text { Hyp } \\ 3(3) p \supset q & \text { Hyp } \\ 4(4) q \supset r & \text { Hyp } \\ 3,4(5) p \supset r & 3,4 \text { NPC }\end{array}$




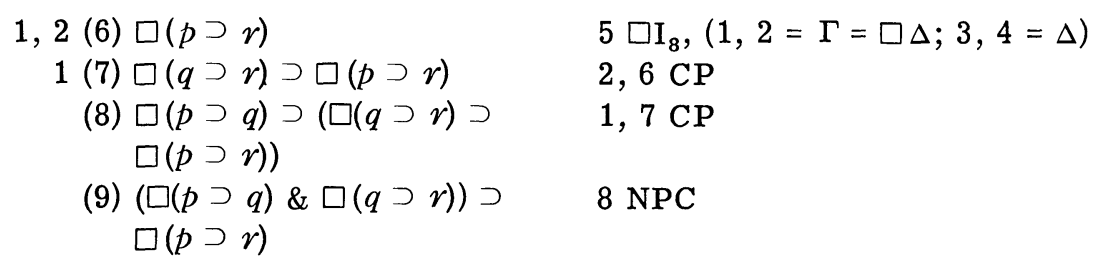

Again, in a step of $\square I_{8}$ there are hypotheses discharged, where $\Delta$ is not empty. The restriction that only NPC rules be used in the subsidiary deduction is easily checked. Rule $\left(\mathrm{a}^{\prime}\right)$ is $\square \mathrm{I}_{8}$ where $\Delta$ is empty. Finally, rule $\left(\mathrm{b}^{\prime}\right)$ is obtained from (i) $(p \equiv q) \supset(-p \equiv-q)$; (ii) $(p \equiv q) \supset((r \supset p) \equiv$ $(r \supset q))$; (iii) $(p \equiv q) \supset((p \supset r) \equiv(q \supset r))$; and (iv) the rule $\square \mathrm{S}_{3}$, which is a counterpart of the rule $\left(\mathrm{Gb}^{\prime}\right)$ of $[16]$.

Rule $\square S_{2}$ is just rule (b). Thus, it suffices for showing the deductive equivalence of NS2 and S2 to show that $\square \mathrm{I}_{8}$ for NS2 is deductively equivalent to $\left(\mathrm{a}^{\prime}\right)$ for $\mathrm{S} 2$ and $\left(1^{\prime}\right)$ in the presence of NPC, $\square \mathrm{E}_{1}$, and $\square \mathrm{S}_{2}$ (or PC, (2), and (b)). Where $\Delta$ is not empty the proof that $\square \mathrm{I}_{8}$ for NS2 holds for S2 is the same as that given for S1. Of course, where $\Delta$ is empty, $\square I_{8}$ is just ( $\left.a^{\prime}\right)$ for S2. Conversely, $\left(1^{\prime}\right)$ is proven in NS2 by the same proof as that given in NK except that step (6) is now justified by $\square I_{8}$ rather than by $\square I_{1}$. Since the subsidiary deduction from steps (3) through (5) of that proof is by NPC alone the restrictions of $\square \mathrm{I}_{8}$ are met. Finally, $\left(\mathrm{a}^{\prime}\right)$ for $\mathrm{S} 2$ is the special case of $\square I_{8}$ for NS2 where $\Delta$ is empty. Hence, NS2 and S2 are deductively equivalent.

It is obvious that axiom (1) and rule $\square S_{1}$ are deductively equivalent in the presence of NPC or PC with the deduction theorem, just as (2) and $\square \mathrm{E}_{1}$ are. Also, in $\mathrm{S} 3, \square \mathrm{I}_{8}$ for NS3 holds where $\Delta$ is empty since it is then just $\left(a^{\prime}\right)$ for S3. Where $\Delta$ is not empty, the proof that $\square I_{8}$ holds for S3 is the same as that for $\mathrm{S} 1$, since $\left(1^{\prime}\right)$ is derivable in S3. Conversely, in NS3 $\left(\mathrm{a}^{\prime}\right)$ for S3 holds as a special case of $\square \mathrm{I}_{8}$ where $\Delta$ is empty; (1) and (2) hold by virtue of $\square E_{1}$ and $\square I_{8}$ for NS3. Thus we obtain the deductive equivalence of NS3 and S3.

The relationships of $\mathrm{S} 1-\mathrm{S} 3$ to each other and to $\mathrm{S} 4$ and $\mathrm{T}$ are easily observed here. The progression from NS1 to NS3 is one of liberalizing restrictions on introducing ' $\square$ ' into a proof. NS2 contains NS1, and NS3 contains NS2 in virtue of the derivability of $\square S_{2}$ from $\square S_{1}$. Further, $\square I_{8}$ for NS3 and $\square \mathrm{S}_{1}$ are derivable from $\square \mathrm{I}_{4}$; so, NS4 contains NS3. However, $\square \mathrm{I}_{8}$ for NS3 is not derivable from $\square I_{1}$ since for NS3 $\Lambda$ of $\square I_{8}$ contains (1) which is not provable from $\square I_{1}$. So NT does not contain NS3. Further NS3 does not contain NT since $\square I_{1}$ is not derivable from $\square I_{8}$ for NS3 given the restrictions for the case where $\Delta$ is empty. But $\square I_{8}$ for NS2 is easily derivable from $\square \mathrm{I}_{1}$ and $\square \mathrm{E}_{1}$, since both (1') and (2) are provable by these rules. So NT contains NS2. Thus the relations of these systems noticed by Lemmon, p. 180 of [6], are obvious when the systems are given natural deduction formalizations also. Moreover, if we further restrict the introduction of ' $\square$ ' by replacing $\square I_{8}$ in $N S 1$ by $\square I_{9}$, we then get a system deductively equivalent to S0.5. Thus we have:

S0.5: PC; $\left(\mathrm{a}^{\prime \prime}\right) ;\left(1^{\prime}\right) ;(2)$. NS0.5: NPC; $\square \mathrm{I}_{9} ; \square \mathrm{E}_{1}$. 
In S0.5, NPC and $\square \mathrm{E}_{1}$ hold by PC, the deduction theorem, and (2) as usual. $\square \mathrm{I}_{9}$ is obtained as follows: if $\Delta$ is empty then we have just a special case of rule $\left(a^{\prime \prime}\right)$ since all theorems of NPC are instances of tautologies. If $\Delta$ is not empty, then proof is the same as that given for $\square \mathrm{I}_{8}$ of NS1 for S1, citing $\left(a^{\prime \prime}\right)$ in place of $\left(a^{\prime}\right)$. Conversely, in NSO.5 we have (2) by $\square \mathrm{E}_{1}$ as usual. $\left(a^{\prime \prime}\right)$ is $\square I_{9}$ where $\Delta$ is empty, and $\left(1^{\prime}\right)$ follows by the same proof as that in $\mathrm{NK}$, noting that at line (6) rule $\square \mathrm{I}_{9}$ would be cited correctly since all of the restrictions of that rule are met by the previous proof.

In the same manner we can construct corresponding natural deduction systems for the families of $\mathrm{E}-$ and $\mathrm{D}$-systems. The deductive equivalence of $\square \mathrm{E}_{1}$ with (2) in the presence of NPC or PC has already been noted. Also, in the presence of $\mathrm{PC}$ and (2) either (1) or (1') together with (Eb) permits us to derive $\square \mathrm{I}_{2}$ as follows. Suppose that $A_{1}, \ldots, A_{n} \vdash B$. By PC we have $\vdash A_{1} \supset\left(A_{2} \supset\left(\ldots \supset\left(A_{n} \supset B\right) \ldots\right)\right.$. By $(\mathrm{Eb})$ we have $\vdash \square A_{1} \supset \square\left(A_{2} \supset(\ldots \supset\right.$ $\left.\left(A_{n} \supset B\right) \ldots\right)$, and so repeated use of $\left(1^{\prime}\right)$ and $\mathrm{PC}$ yields $\vdash \square A_{1} \supset\left(\square A_{2} \supset\right.$ $\left(\ldots \supset\left(\square A_{n} \supset \square B\right) \ldots\right)$. Thus given a proof of $\square A_{1}, \ldots, \square A_{n}$ from $\Gamma$, this theorem enables us to prove $\Gamma \vdash \square B$. Since $\left(1^{\prime}\right)$ is derivable from (1), given (2), the above proof holds for systems having axiom (1) in place of $\left(1^{\prime}\right)$, provided that they have (2). Conversely, $\square I_{2}$ yields $\left(1^{\prime}\right)$ by the same proof given in NK and yields (1) in the presence of the additional $\square S_{1}$. (Eb) results as follows: suppose that $\vdash A \supset B$. Then we use the following proof scheme for any system containing NPC and $\square I_{2}$ :

$\begin{array}{ll}1 \text { (1) } \square A & \text { Hyp } \\ 2 \text { (2) } A & \text { Hyp } \\ \text { (3) } A \supset B & \text { Theorem by supposition } \\ 2 \text { (4) } B & 2,3 \mathrm{MP} \\ 1 \text { (5) } \square B & 1,2,4 \square \mathrm{I}_{2} \\ \text { (6) } \square A \supset \square B & 1,5 \mathrm{CP}\end{array}$

The restrictions on $\square I_{2}$ are met in this proof scheme since line (3) is not a hypothesis, though it is a premise. Thus the fact that no line of the proof scheme contains $\square(A \supset B)$ is not a problem because $A \supset B$ is not in $\Delta$ anyway.

The above considerations yield natural deduction systems NE2 and NE3 deductively equivalent to E2 and E3 respectively:
$\mathrm{E} 2: \mathrm{PC} ;(\mathrm{Eb}) ;\left(1^{\prime}\right) ;(2)$.
NE2: NPC; $\square \mathrm{I}_{2} ; \square \mathrm{E}_{1}$.
E3: $\mathrm{PC}$; $(\mathrm{Eb}) ;(1) ;(2)$.
NE3: NPC; $\square \mathrm{I}_{2} ; \square \mathrm{E}_{1} ; \square \mathrm{S}_{1}$.

If we restrict the wffs $A, A_{1}, \ldots, A_{n}, B$ of the previous paragraph to wffs of PC (NPC), i.e., wffs not containing modal operators, we have a proof of the deductive equivalence of $\left(E b^{\prime}\right)$ and $\left(1^{\prime}\right)$ with $\square I_{3}$. Thus we also have shown the deductive equivalence of:
E1: PC; $\left(\mathrm{Eb}^{\prime}\right) ;\left(1^{\prime}\right) ;(2)$.
NE1: NPC; $\square \mathrm{I}_{3} ; \square \mathrm{E}_{1}$.

Corresponding to the remaining E-systems we have:

E4: PC; (Eb); (1'); (2); (4).

E5: PC; (Eb); $\left(1^{\prime}\right) ;(2) ;(5)$.
NE4: NPC; $\square \mathrm{I}_{5} ; \square \mathrm{E}_{1}$.

NE5: $\mathrm{NPC} ; \square \mathrm{I}_{7} ; \square \mathrm{E}_{1}$. 
By a proof similar to that deriving $\square \mathrm{I}_{4}\left(\square \mathrm{I}_{6}\right)$ from (a) and (4) ((a) and (5)) we can derive $\square \mathrm{I}_{5}\left(\square \mathrm{I}_{7}\right)$ from (Eb) and (4) ((Eb) and (5)) in the presence of $\left(1^{\prime}\right)$. Conversely, (4) holds in NE4 by the same proof given in NS4, and (5) is proved in NE5 by the same proof given in NS5, since both (i) - $\square--p \supset$ $-\square p$ and (ii) $-\square p \supset \diamond-p$ are easily derived as theorems of NE5. (1') is provable in NE4 and NE5 as follows, in the latter system citing $\square I_{7}$ at line (6):

$\begin{array}{rl}1(1) \square(p \supset q) & \text { Hyp } \\ 2(2) \square p & \text { Hyp } \\ 1(3) p \supset q & 1 \square \mathrm{E}_{1} \\ 2(4) p & 2 \square \mathrm{E}_{1} \\ 1,2(5) q & 3,4 \mathrm{MP} \\ 1,2(6) \square q & 5 \square \mathrm{I}_{5} \\ 1(7) \square p \supset \square q & 2,6 \mathrm{CP} \\ (8) \square(p \supset q) \supset(\square p \supset \square q) & 1,7 \mathrm{CP}\end{array}$

This proof is also available in both NS4 and NS5.

Systems D1 and D2 differ from E1 and E2 only in having the weaker axiom ( $\left.2^{\prime}\right)$ in place of (2). In the presence of PC or NPC rule $\square E_{2}$ and ( $\left.2^{\prime}\right)$ are deductively equivalent just as $\square E_{1}$ and (2) are. Also, the deductive equivalence of $\square I_{3}$ with $\left(E b^{\prime}\right)$ and $\left(1^{\prime}\right)$ and of $\square I_{2}$ with $(E b)$ and (1') does not require that (2) or $\square \mathrm{E}_{1}$ be present, though the deductive equivalence of (Eb) and (1) with $\square I_{2}$ did require the presence of (2) or $\square E_{1}$. Thus, there are deductively equivalent natural deduction systems for D1 and D2, viz.:
D1: PC; $\left(\mathrm{Eb}^{\prime}\right) ;\left(1^{\prime}\right) ;\left(2^{\prime}\right)$.
$\mathrm{D} 2$ : $\mathrm{PC} ;(\mathrm{Eb}) ;\left(1^{\prime}\right) ;\left(2^{\prime}\right)$.
ND1: $\mathrm{NPC} ; \square \mathrm{I}_{3} ; \square \mathrm{E}_{2}$.
ND2: NPC; $\square \mathrm{I}_{2} ; \square \mathrm{E}_{2}$.

The remaining $D^{-}$-systems have both the rule (D) and $\left(2^{\prime}\right)$ in place of (2) of the corresponding E-systems. It is easily shown that (D) and $\left(2^{\prime}\right)$ are deductively equivalent to $\square \mathrm{E}_{3}$ in the presence of PC. Further, for D3, (D) and (1) permit derivation of $\left(1^{\prime}\right)$. So the previously shown deductive equivalence of (Eb) and (1) with $\square \mathrm{I}_{2}$ and $\square \mathrm{S}_{1}$ continues to hold, now in the presence of $(D)$ and $\left(2^{\prime}\right)$ or $\square E_{3}$. This yields the deductive equivalence of D3: PC; (Eb); (D); (1); (2'). ND3: NPC; $\square \mathrm{I}_{2} ; \square \mathrm{E}_{3}$.

Because of the lack of (2) in D4 and D5, the corresponding systems ND4 and ND5 require the hybrid rules $\square \mathrm{I}_{10}$ and $\square \mathrm{I}_{11}$, which allow proof of $\left(1^{\prime}\right)$ and $(\mathrm{Eb})$, in the absence of $\square \mathrm{E}_{1}$, as well as (4) and (5) respectively. We have:

D4: PC; (Eb); (D); (1'); (2'); (4). ND4: NPC; $\square \mathrm{I}_{10} ; \square \mathrm{E}_{3}$.

D5: PC; (Eb); (D); (1'); (2'); (5). ND5: NPC; $\square \mathrm{I}_{11} ; \square \mathrm{E}_{3}$.

For the deductive equivalence of $\mathrm{D} 4$ and ND4, it suffices to show that (Eb), $\left(1^{\prime}\right)$ and (4) yield $\square I_{10}$ and that $\square I_{10}$ yields (Eb), ( $\left.1^{\prime}\right)$ and (4) in ND4. $\square I_{10}$ is derivable for $\mathrm{D} 4$ as follows: let $A_{1}, \ldots, A_{n} \vdash B$ in $\mathrm{D} 4$ be given. By the deduction theorem for $\mathrm{D} 4: \vdash A_{1} \supset\left(\ldots \supset\left(A_{n} \supset B\right) \ldots\right)$ in D4. By $(\mathrm{Eb}),\left(1^{\prime}\right)$, and $P C$ :

$$
\text { (a) } \vdash \square A_{1} \supset\left(\ldots \supset\left(\square A_{n} \supset \square B\right) \ldots\right. \text {. . in D4. }
$$


Hence, (i) given a proof of $\square A_{1} \& \ldots \& \square A_{n}$ from $\Gamma$ (a) yields a proof of $\Gamma \vdash \square B$ in $\mathrm{D} 4$; (ii) if each $A_{i}(1 \leq i \leq n)$ is $\square C_{i}$ for some wff $C_{i}$ then, by the theorem of $\mathrm{D} 4$ : $\square C_{i} \supset \square \square C_{i}$, we have $\vdash A_{i} \supset \square A_{i}$ in $\mathrm{D} 4$. So, by $\mathrm{PC}$ : $\vdash A_{1} \supset\left(\ldots \supset\left(A_{n} \supset \square B\right) \ldots\right)$ in D4. Thus, $A_{1}, \ldots, A_{n} \vdash \square B$ in D4. Conversely, in ND4 $\left(1^{\prime}\right)$ is derivable by either the proof in NK or that in NE4, citing $\square \mathrm{I}_{10}$ at line (6) in either case. (4) is easily derived by rules Hyp, $\square \mathrm{I}_{10}$, and CP. Finally, (Eb) follows by the previous proof of (Eb) for NE2, using $\square I_{10}$ rather than $\square I_{2}$. The deductive equivalence of (Eb), (1'), and (5) with $\square I_{11}$ is shown similarly, and so the proof is omitted.

The containment relations pointed out by Lemmon between S- E- and D-systems are easily shown on the new rules here. For any S-system, the corresponding E-system is obtained by restricting the conditions for introducing and distributing ' $\square$ ' in proofs. The corresponding D-system is obtained by also restricting the conditions for eliminating ' $\square$ ' from proofs.

3. There is always a greater risk of error in drawing philosophical morals about a concept from a set of syntactic rules for a formal system than there is in drawing such morals from a semantic model of that system. Nevertheless, the following are drawn. A formal system $S$ is, under interpretation, a system of reasoning in which one employs the concepts formalized. Given a formal system $S$, it is plausible to interpret $A_{1}, \ldots$, $A_{n} \vdash B$ in $S$ as " $B$ is warranted by $S$ as an assertion on the basis of $A_{1}, \ldots$, $A_{n}$." If one is warranted by $S$ to assert $B$ given certain hypotheses, then a $\square$ introduction rule states the further conditions, if any, required of one's hypotheses under which $S$ would also warrant the assertion of $\square B$. For example, interpreting ' $\square$ ' as 'Jones knows that', $\square \mathrm{I}_{2}$ warrants that Jones knows that $B$ provided that he knows each of $A_{1}, \ldots, A_{n}$ and that $A_{1}, \ldots$, $A_{n} \vdash B$ in the system of reasoning considered. Both E2 and E3 (or NE2 and NE3) are plausible formalizations for ' $k$ now' restricted to individuals. However, under this interpretation $\square I_{5}$ would allow us to infer that Jones knows anything which follows from propositions to the effect that he knows certain things to be true. This perhaps makes E4 or NE4 unsuitable as a formalization of a given person's knowledge.

On the other hand, the fact that a proposition $B$ follows from propositions stating that certain things have been verified constitutes a sort of verification of $B$. So NE4, with $\square \mathrm{I}_{5}$, might provide a plausible formalization of the concept 'it is verified that'. Further, $\square \mathrm{I}_{5}$ provides that nothing is verified simply by being a theorem of logic. Thus the concept formalized here would be applicable only to propositions contingent relative to NE4 or E4. However, the choice of an interpretation for a system of modal logic is difficult to make without considering set theoretic models for the system. These cannot be taken up here.

\section{REFERENCES}

[1] Curry, H. B., A theory of formal deducibility, University of Notre Dame Press, Notre Dame (1950). 
[2] Fitch, F. B., Symbolic logic: an introduction, Ronald Press Co., New York (1952).

[3] Kanger, Stig, Provability in logic, Almquist and Wiksell, Uppsala (1957).

[4] Kleene, S. C., Introduction to metamathematics, Van Nostrand, Princeton (1952).

[5] Lemmon, E. J., Beginning logic, Nelson, London (1965).

[6] Lemmon, E. J., "New foundations for Lewis modal systems," The Journal of Symbolic Logic, vol. 22 (1957), pp. 176-186.

[7] Matsumoto, Kazuo, "Decision procedure for modal sentential calculus S3," Osaka Mathematical Journal, vol. 12 (1960), pp. 167-175.

[8] Moh Shaw-Kwei, "The deduction theorems and two new logical systems," Methodos, vol. 2 (1950), pp. 56-75.

[9] Ohnishi, Masao, "Gentzen decision procedures for Lewis's systems S2 and S3," Osaka Mathematical Journal, vol. 13 (1961), pp. 125-137.

[10] Ohnishi, Masao, and Kazuo Matsumoto, "Gentzen method in modal calculi," Osaka Mathematical Journal, vol. 9 (1957), pp. 113-130.

[11] Ohnishi, Masao, and Kazuo Matsumoto, "Gentzen method in modal calculi, II," Osaka Mathematical Journal, vol. 11 (1959), pp. 115-120.

[12] Prior, A. N., Formal logic, Oxford University Press, Oxford, second edition (1961).

[13] Robbin, Joel W., Mathematical logic, W. A. Benjamin, New York (1969).

[14] Routley, Richard, "The decidability and semantical incompleteness of Lemmon's system S0.5," Logique et Analyse, vol. 11 (1968), pp. 413-421.

[15] Suppes, Patrick, Introduction to logic, Van Nostrand, Princeton (1957).

[16] Zeman, J. Jay, "Lemmon-style bases for the systems $\mathrm{S} 1^{\circ}-\mathrm{S} 4^{\circ}$," The Journal of Symbolic Logic, vol. 33 (1968), pp. 458-461.

Bradley University

Peoria, Illinois 